\title{
Estudio descriptivo de la evolución de los modelos de gestión operativa y estratégica de las empresas manufactureras locales y de capital extranjero localizadas en Puebla - México
}

Descriptive study of the evolution of the operational and strategic management models of local manufacturing enterprises and foreign capital located in Puebla - Mexico

Recibido: enero 11 de 2017 | Revisado: marzo 10 de 2017 | Aceptado: abril 15 de 2017

JUVENCIO ROLDÁN ${ }^{\mathrm{I}}$ JAVIER MÉNDEZ ${ }^{2}$

Alejandro Olvera ${ }^{3}$

Airam Ledezma ${ }^{4}$
1 Benemérita Universidad Autónoma de Puebla. México juvencioroldan@gmail.com

2 Benemérita Universidad Autónoma de Puebla. México fimendez@itesm.mx

3 Benemérita Universidad Autónoma de Puebla. México alejandro.olvera@vorreo.buap.mx

4 Benemérita Universidad Autónoma de Puebla. México airamledezma@hotmail.com

\section{Resumen}

Las empresas manufactureras globales han evolucionado sus modelos de negocios en cuatro factores esenciales: procesos flexibles en lugar de procesos rígidos, competitividad colaborativa por competitividad depredadora, de explotación de recursos baratos a basadas en intangibles y de organizaciones que hacen trabajo burocrático a organizaciones orientadas al trabajo estratégico, pero el sector manufacturero de origen local, de la ciudad de Puebla, no ha dado dicho salto evolutivo y se encuentran rezagado en los cuatro factores. Como complemento, se asume que existen dos elementos pivotantes, que contribuyen a la evolución de los otros factores en la forma más económica, estos son: relaciones de confianza y organizaciones basadas en tecnologías de la información. En el estudio se encuesta a los directores y gerentes de 10 empresas de origen extranjero con presencia global y con plantas en la ciudad de Puebla, y a ocho directores y socios de empresas manufactureras de capital de origen poblano. Los resultados de la investigación evidencian efectivamente el rezago en algunos factores de los modelos de negocios de las empresas manufactureras de origen local, así como se demuestra la contribución de los elementos pivotantes a la evolución económica de los factores esenciales.

Palabras clave: evolución económica, modelo de negocios, empresas manufactureras

\section{Abstract}

Global manufacturing companies have evolved their business models on four essential factors: from rigid to flexible processes, from predatory competitiveness to collaborative competitiveness, from cheap resources exploitation to the use of intangible assets, and from organizations doing bureaucratic work to campanies doing strategic work instead. Nevertheless, the domestic manufacturing companies, that means, companies whose owners are from Puebla City, have not given the 
mentioned evolutionary leap and stay laggard in the mentioned four factors. In addition, it is assumed that two pivotant elements that contribute to the others economical evolution factors exist, and these are: business relationships based on trust, and information technologies based organizations. For the research, were surveyed CEO's and chiefs of 10 global manufacturing companies with facilities in Puebla City, and CEO's and owners of eight manufacturing companies with Puebla capital origin? The results of the research make evident the domestic manufacturing companies business model's lag in some issues, as well as demonstrates the contribution of the pivoting elements to the economical evolution of the essential factors.

Key words: economic evolution, business model, manufacturing companies.

\section{Introducción}

Competir en la reñida arena global es un reto que las empresas manufactureras mexicanas deberán asumir si aspiran a permanecer haciendo negocios en este siglo de la globalización. La competitividad es una característica que se fortalece de diversos factores internos y externos a las empresas y a las regiones, y que, en este artículo, se revisarán y comprobarán partiendo de algunas hipótesis respaldadas por la literatura correspondiente.

A lo largo de la historia de la humanidad, la geografía y el clima jugaron un rol clave en la capacidad de las naciones y sus regiones para generar riqueza, como lo evidencia en el primer capítulo de su libro "La riqueza y la pobreza de las naciones" el historiador de Harvard, David Landes. En este, relata cómo hay naciones localizadas, en ciertas latitudes, a las que nunca favoreció su clima, ni su ubicación para producir alimentos de alta calidad y bajo costo, mientras que otras regiones se han visto siempre, ampliamente, favorecidas por dichos factores "casuales" (Landes, 2008).
El desarrollo tecnológico postrenacentista, fortalecido por la revolución industrial, trajo consigo nuevas ventajas competitivas a las naciones que se subieron primero a dicho movimiento. La tecnología otorgó la posibilidad de acceder a más recursos, dispersos a lo largo $\mathrm{y}$ ancho del orbe, con mejores costos y en tiempos más cortos. Las ventajas de la tecnología, que durante finales del siglo XIX y la primera mitad del XX estuvo restringida a sus creadores, se reflejaron en el poder militar, subyugando y obteniendo el máximo provecho de aquellas regiones que no contaban con esta. Sobre estos principios se escribió la historia del colonialismo y del imperialismo en la segunda parte del segundo milenio de la era cristiana.

Las ventajas competitivas de la geografía y el clima, así como de la propiedad de la tecnología no se aprovecharon, exclusivamente, en poderío militar, sino también en la producción más económica de alimentos, mejores niveles de salud y educativos en las respectivas poblaciones que generaron así círculos virtuosos. 
No obstante, la segunda mitad del siglo XX y un desarrollo más acelerado de la tecnología, planteó un cambio radical de paradigma. Evidencias de cómo el factor geográfico perdió importancia, están en la forma en la que el pueblo judío transformó su desierto en un lugar habitable y en el que hoy se genera enorme riqueza basada en la creación de conocimiento. Cómo los japoneses lograron superar los niveles de riqueza de muchos países europeos con larga trayectoria y de los mismos Estados Unidos. Cabe destacar casos como el de la próspera ciudad de Monterrey en México, en una región sumamente árida o Singapur y Hong Kong, países muy pequeños y sin acceso a vastos recursos naturales.

Son muy diversos los estudios en los que se han tratado de explicar los factores que han empujado la competitividad de algunas regiones y sus empresas, o viceversa, por los que otras regiones y sus empresas son incapaces de mejorar, competir en la arena global y así impulsar la prosperidad de sus habitantes. Uno de los estudiosos de la competitividad más reconocidos mundialmente, es el del profesor de Harvard, Michael Porter (1999), autor del libro "Estrategia Competitiva", quien entre muchas otras afirmaciones célebres sostiene que la competitividad de un país o una región es el resultado de la productividad de las empresas de la región. En esta investigación, se alude a esta afirmación como base para encontrar aquellas razones inherentes al desempeño interno de las compañías manufactureras como impulsores de la competitividad nacional, contrariamente a la búsqueda de soluciones en factores externos, fuera del alcance de las empresas que, en muchas ocasiones pueden interpretarse como búsqueda de chivos expiatorios, y no de intenciones reales por mejorar. Más aún, y retomando otra vez a Michael Porter, en su documento "Índice de Innovación", se considera que las ventajas competitivas, basadas en tecnología son efímeras y sujetas a la conducción de otros factores que están más relacionados con la cultura y su relación con los modelos de negocios. Así que se comienza esta investigación acotando ambos conceptos, para luego describir y sustentar cada uno de los factores que conforman el modelo de evolución que se propone comprobar en el trabajo.

La industria manufacturera de capital con origen poblano está desapareciendo gradualmente, como consecuencia de su baja competitividad en los mercados globalizados. Esta situación podría reflejarse en un deterioro constante de los niveles de empleo y bienestar de los habitantes de la región. Aunque en el presente estudio nos ocuparemos de empresas manufactureras localizadas en la ciudad de Puebla por razones de presupuesto y tiempo, la problemática es de alcance nacional, como lo confirma el siguiente texto del Dr. Enrique Dussel Peters de la Facultad de Economía de la UNAM: "El aparato productivo en México, particularmente, el manufacturero, ha tenido profundas dificultades para insertarse en el mercado internacional y para mantener su posición en el mercado interno. La manufactura presenta enormes dificultades con el fin de incrementar su valor agregado desde el proceso de apertura a finales de la década de los ochenta, así como una de las principales paradojas de la industrialización orientada hacia las exportaciones. Para incrementar su PIB, la manufactura requiere de cada vez mayo- 
res incrementos de insumos importados; su magnitud afecta, sustancialmente, la balanza comercial, de cuenta corriente y la macroeconomía en su conjunto. Desde esta perspectiva, diversos estudios señalan importantes rupturas de cadenas de valor desde los noventa y unas enormes dificultades para endogeneizar procesos por parte del sector manufacturero industrial.

Parte de la problemática anterior una orientación exportadora con base en importaciones temporales, con alto contenido importado y su creciente falta de competitividad- se hace latentes, en la competencia actual con la República Popular China, en el mercado interno y, en el principal mercado exportador mexicano es los Estados Unidos. A nivel de tamaño de las empresas, el sector manufacturero se ha rezagado con respecto al resto de la economía durante 1999-2004. Destaca, particularmente, la pérdida absoluta de empleo y establecimientos. Sin el objetivo de abarcar la riqueza de este debate y del cuadro, sobresalen tres tendencias adicionales:

a) La manufactura, pierde en forma significativa en todos los rubros analizados: establecimientos, empleo y valor agregado bruto; en el caso de este último, por ejemplo, la participación de la manufactura cae $37.73 \%$ del total en 1999 a $28.24 \%$ en 2004.

b) Las microempresas -todas aquellas con 10 o menos ocupados- participan con $38.33 \%$ del empleo nacional.

c) En la manufactura, han sido las grandes empresas, es decir, todas aquellas con más de 250 ocupados, las que se han beneficiado en términos de empleo generado y su participación se incrementó de $48.27 \%$ en 1999 a $52.27 \%$ en 2004 .

A nivel microeconómico, es importante destacar al menos tres aspectos:

a) La dificultad de generar empleo - y su expulsión desde 2000- por parte de la manufactura (y la especialización del sector exportador en importaciones temporales para su exportación).

b) Diversas tendencias de las empresas, según su tamaño, sus dificultades con respecto al registro y la "tramitología", $\mathrm{y}$

c) Las dificultades de las empresas en México en general, y en particular en la manufactura, para generar empleo formal como resultado de procesos basados en importaciones temporales.

Buena parte de la problemática señalada anteriormente -la falta de endogeneidad y de integración con el resto del aparato productivo- se refleja en la falta de generación de empleos por parte de la manufactura mexicana: si bien la manufactura se caracteriza por un, relativamente alto, nivel de formalización -en términos de registro ante el Instituto Mexicano del Seguro Social -con respecto al resto de la economía, el sector no solo no ha podido generar empleo, sino que se encuentre en un profundo proceso de crisis desde diciembre de 2000 y hasta 2005 la manufactura perdió $15.3 \%$ de su empleo.

Diversos autores han destacado que si bien las exportaciones se han convertido en el motor de crecimiento manufacturero - y de la economía en su conjunto - este realiza procesos relativamente primitivos, con bajo valor agregado y que, 
adicionalmente, ha destruido encadenamientos existentes hacia delante y atrás; no obstante, los incrementos observados en la productividad [como resultado de la disminución del empleo y de un PIB constante durante 2000-2005, por ejemplo" (Dussel, 2006)].

Las empresas manufactureras de capital de origen local (ciudad de Puebla y municipios aledaños), no destacan en los mercados globales, es decir, no ostentan ninguna forma de liderazgo en los mercados de otros países.

Dicho liderazgo o posición destacada, implica que han sido capaces de replicar sus sistemas de producción, en otras regiones del mundo, instalando sus propias plantas o a través de alianzas estratégicas, manteniendo los mismos estándares de calidad, beneficios de adopción de tecnología y modelo de negocios y obtener así los beneficios de cubrir mayores extensiones territoriales sin incrementar los costos de transportación de producto terminado, además de mejorar su posicionamiento, en las mismas regiones, al generar empleos y riqueza en las mismas. Dichos procesos de replicación exitosa se manifiestan, a su vez, en enormes volúmenes de facturación, número de empleados contratados, indicadores de rentabilidad y prestigio de marca, que les vale el reconocimiento por diversos rankings.

También son reconocidas por el nivel de satisfacción de sus empleados y sus innovaciones, lo cual puede interpretarse como una causa y efecto simultáneos de sus posiciones exitosas. Esta situación se evidencia en los rankings de diversas publicaciones de prestigio internacional como: Las 500 de Fortune (Internacional) elaborado y publicado por la revista de negocios Fortune, las 500 de Expansión del 2000 al 2008, (Nacional), elaborado y publicado por la revista mexicana de negocios Expansión, ambas basadas en datos duros, tales como:

- Monto anual de ventas en dólares

- Número de empleados

- Utilidades operativas netas

- Relación de ganancias por activos

Tales índices son tomados en cuenta tanto por el World Economic Forum en su Competitiveness Index como por el Instituto Mexicano para la Competitividad (IMCO) y el EGAD (Escuela de Graduados en Administración y Políticas Públicas del Tecnológico de Monterrey) en el Reporte de Competitividad Estatal México 2016, que cuenta con un vasto soporte metodológico. En el documento del IMCO y EGAD, las empresas del Estado de Puebla se ubican muy por debajo de las de varios Estados de la República en varios factores, tales como:

- Empresas en 500 de expansión

- Empresas certificadas con ISO 9000

- Coeficientes de invención

- Número de empresas manufactureras grandes

Más aún, en dicho documento, se analizan los pesos e importancias de cada factor, y el de las 500 de expansión aparece con un peso de 0.9 y por lo tanto, considerada como muy importante. (IMCO,2016)

Otro estudio en el que no figura ninguna empresa manufacturera poblana, y es elaborado cada ańo desde 2004, es el de 
las empresas líderes de México, realizado por Hay Group y HSM. Hay Group es una prestigiosa firma global de consultoría en gestión empresarial que opera desde hace 60 años en el mundo y 40 años en México. HSM es la editora de la revista Gestión de Negocios y organizadora del evento anual Expomanagement.

En el Índice de Competitividad Global 2016 del World Economic Forum, aunque solamente aparece el dato nacional, nuestro país aparece muy mal posicionado en el subíndice del grado de Sofisticación de Negocios (Business Sophistication) en el lugar 57 , que considera aspectos como:

- Redes e industrias de apoyo:

- Cantidad y calidad de los proveedores locales

- Sofisticación de la estrategia y operaciones de las compañías

- Sofisticación de los procesos de producción

- Control de la distribución internacional

- Disponibilidad para delegar autoridad

- Naturaleza de sus ventajas competitivas

- Presencia de la cadena de valor

En otros índices basados en encuestas y no en datos duros, tales como:

- Las empresas con mejores ingresos (The World's Biggest Public Companies, 2017)
- Las más admiradas (Fortune, 2016/)

- The world's most respected companies 2016. Estudio elaborado por Reputation Institute, y en el que aparecen mencionadas cuatro empresas mexicanas, Bimbo en el lugar 9, empresas de Argentina, Brasil, Chile, China, India y otros países desarrollados y en vías de desarrollo.

Tampoco figura ninguna empresa de capital local ubicada en Puebla. Sin embargo, vale la pena mencionar que, en el índice de las mejores empresas para trabajar, encuesta realizada por "Great Place to Work Institute Mexico" aparece la empresa Arvin Meritor, del sector autopartes, de capital de origen estadounidense y que tiene una planta en esta ciudad. Esta es una de las empresas extranjeras en las que se aplicó la encuesta para determinar el grado de evolución de la presente investigación. En ningún ranking, aparece alguna empresa manufacturera de capital local de ningún sector ni tamaño. En el de las empresas líderes de México, figuran empresas mexicanas de capital nacional, pero ninguna tiene su origen en Puebla, algunas de estas son: PEMEX, Grupo Carso, Telmex, América Móvil, CEMEX, FEMSA, BIMBO, Televisa, Minera Peńoles, La Costeña, Jugos del Valle, COMEX, ALPURA, GAMESA y BACHOCO. 


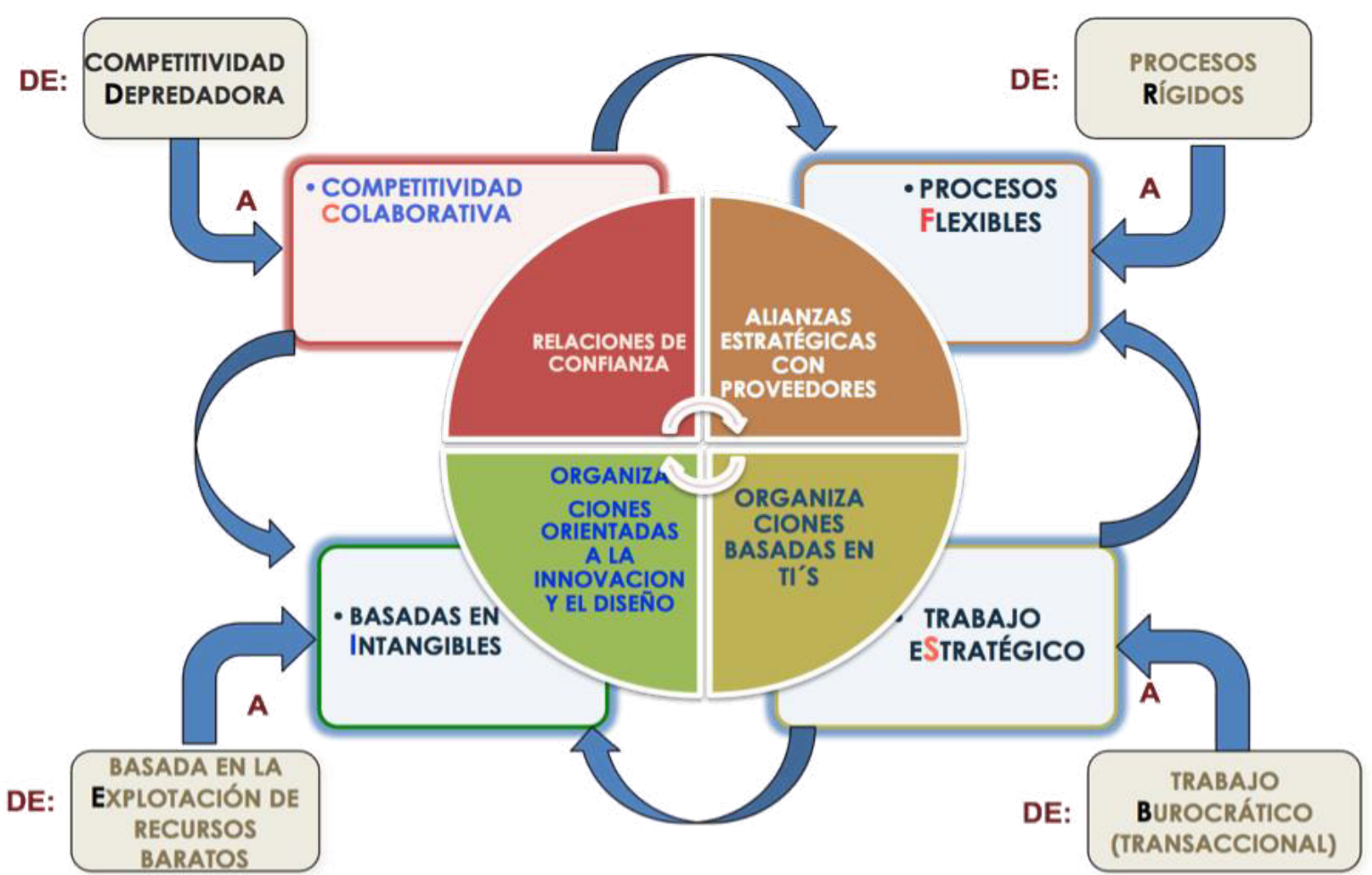

Figura 1. Flujo de economía

Estas listas incluyen a las compañías de mayor presencia, crecimiento y competitividad global, provenientes de países como Brasil, Chile, Argentina, China, India, Turquía, Rusia, Egipto, Hungría, Malasia, Polonia, Indonesia, Tailandia y México. Aparecen ocho compañías mexicanas: América Móvil, CEMEX, GRUMA, FEMSA, Grupo Bimbo, Mexichem y Nemak. Ninguna de origen poblano.

Desafortunadamente y a pesar de la exhaustiva búsqueda, no se encontraron otros rankings nacionales o internacionales de competitividad empresarial en Journals, revistas indexadas u otro tipo de publicación de origen académico que ofrezca mayor soporte al argumento de la baja competitividad de las empresas manufactureras locales. Cabe también preguntarse si en los rigurosos estudios de origen académico tendrían la posibilidad de destacar las empresas locales que no constan en los de círculos no académicos o del periodismo de negocios. Otras posibles referencias de competitividad, pueden ser las de los premios a las mejores empresas manufactureras, tales como:

- Shingo Prize, otorgado por Utah State University

- Premio Nacional de Calidad, promovido por el Gobierno Federal de la República Mexicana

- Premio Nacional de Tecnología

- Premio al Manufacturero del año, por grupo editorial Expansión, y

- Premio Iberoamericano a la Calidad

De los cuales ninguno ha sido otorgado a una empresa manufacturera de origen poblano, como se puede constatar en sus respectivos sitios de Internet. El riesgo de no alcanzar presencia global es que, en un futuro ni siquiera puedan sobrevivir en el mercado local, dada la constante 
penetración de empresas globales en el mercado local.

\section{Cultura y competitividad}

En el modelo, se integran cuatro factores, cada uno de los cuales se estructura de una serie de elementos que permiten la vinculación con los otros. Cada uno de estos factores ha estado presente en las empresas desde sus inicios, como parte de los modelos de negocios y factores culturales propios de la era industrial, pero que durante los últimos 20 años han sufrido un proceso evolutivo dentro del nuevo contexto global. Estos son:

\section{Procesos flexibles}

Evolucionaron de procesos rígidos, altamente estandarizados y soportados, básicamente, sobre economías de escala y procesos de flujo continuo. Hoy, que la competencia global demanda una oferta diferenciada, basada en la diversificación, es imprescindible contar con un sistema de producción flexible con el que se produzca una alta diversidad y se responda velozmente a los cambios bruscos de la demanda, sin elevar los costos operativos. Los procesos flexibles yacen, por una parte, en un importante componente tecnológico; sin embargo, existen muchos factores que la tecnología por sí sola no soluciona, sino que son el resultado de todo un cambio de cultura organizacional como se comprueba, más adelante, en este mismo capítulo. El elemento que se considera, en este artículo, como el vinculador con otros factores es el de las Alianzas Estratégicas con Proveedores, dada la importancia que ha alcanzado, en los últimos 20 años, sobre otros elementos. (Figura 1)

\section{Competitividad colaborativa}

Evoluciona de la tradicional Competencia Depredadora del siglo pasado y que, en muchas organizaciones sigue vigente. Como se demostró, la colaboración entre empresas marcó un nuevo paradigma y fue un factor de carácter cualitativo digno de estudio en las empresas globales y locales situados en nuestra ciudad de Puebla. Se considera como el elemento pivotante en las Relaciones de confianza. La actitud de confianza implica todo un cambio cultural que mueve a otros factores evolutivos.

\section{Basadas en intangibles}

A diferencia de muchas empresas tradicionales que basaron su competitividad en la explotación de recursos humanos o naturales baratos que se evidencia, hoy, en el valor de las empresas globales en los que se basa los activos intangibles, tales como: patentes, licencias, marcas, recursos humanos, cultura organizacional, habilidades de los empleados, modelo de negocios, alianzas estratégicas, diseño, y algunos más, que en equipos, mano de obra barata o recursos naturales de bajo costo. El elemento de integración en este caso, es la Organización Orientada hacia la innovación creativa y al diseño.

\section{Organizaciones diseñadas para el tra- bajo estratégico}

Evolucionan de organizaciones creadas, originalmente, para efectuar trabajo burocrático o transaccional, necesario antes de la aparición y desarrollo de las Tecnologías de la Información como se demuestra a partir de algunos estudios, 
las TI's otorgan nuevas oportunidades a las organizaciones para reestructurarse y orientar el talento de su gente hacia un trabajo enfocado en la estrategia, que les permita crear procesos más flexibles $\mathrm{y}$ fortalecer sus intangibles. El elemento pivotante con otros factores es la organización diseñada para operar basada en Tecnologías de la Información.

\section{Análisis cuantitativo}

En este estudio se procedió a efectuar una depuración, basada en la significan-

Tabla 1

Resultados del modelo

\section{Resultados preliminares (a)}

\begin{tabular}{|cl|c|c|c|}
\hline & Tipo de empresa & $\mathrm{N}$ & Mean Rank & Sum of Ranks \\
\hline modelo1 & Local & 8 & 6.56 & 52.50 \\
& Global & 10 & 11.85 & 118.50 \\
& Total & 18 & & \\
modelo1p & Local & 8 & 6.50 & 52.00 \\
& Global & 10 & 11.90 & 119.00 \\
& Total & 18 & & \\
\hline
\end{tabular}

Tabla 2

Resultados preliminares del modelo

Test Statistics (b)

\begin{tabular}{|l|c|c|}
\hline & modelo1 & modelo1p \\
\hline Mann-Whitney U & 16.50 & 16.00 \\
Wilcoxon W & 52.50 & 52.00 \\
Z & -2.09 & -2.13 \\
Asymp. Sig. (2-tailed) & .037 & .033 \\
Exact Sig. [2*(1-tailed & $.034(\mathrm{a})$ & $.034(\mathrm{a})$ \\
Sig.)] & \\
\hline
\end{tabular}

a) Not corrected for ties

b) Grouping Variable: Tipo de empresa 
Tabla 3

Resultados experimentales

\begin{tabular}{|ll|c|c|c|}
\hline & Tipo de empresa & $\mathrm{N}$ & Mean Rank & Sum of Ranks \\
\hline \multirow{5}{*}{ st1 proflex } & Local & 8 & 8.13 & 65.00 \\
& Global & 10 & 10.60 & 106.00 \\
& Total & 18 & & \\
& Local & 8 & 7.50 & 60.00 \\
St3 basintang & Global & 10 & 11.10 & 111.00 \\
& Total & 18 & & \\
& Glocal & 8 & 5.13 & 41.00 \\
& Total & 10 & 13.00 & 130.00 \\
& Local & 18 & & \\
& Global & 8 & 7.44 & 59.50 \\
& Total & 10 & 11.15 & 111.50 \\
& 18 & & \\
\hline
\end{tabular}

Se aprecia que las de capital extranjero ostentan mejores calificaciones que las empresas de origen local; sin embargo,

al aplicar la prueba U Mann Whitney, se obtienen las discriminaciones de cada factor por grupo (Tabla 4).

Tabla 4

Comparación de las variables

\begin{tabular}{|l|c|c|c|c|}
\hline & St1 proflex & st2 compcol & st3 basintang & st4 testrateg \\
\hline Mann-Whitney U & 29.00 & 24.00 & 5.00 & 23.50 \\
Wilcoxon W & 65.00 & 60.00 & 41.00 & 59.50 \\
Z & -0.99 & -1.44 & -3.13 & -1.54 \\
Asymp. Sig. (2-tailed) & 0.320 & 0.150 & .002 & 0.124 \\
Exact Sig. [2*(1-tailed & 0.360 & 0.170 & .001 & 0.146 \\
Sig.)] & & & \\
\hline
\end{tabular}

Los factores st 2 compcol (competitividad colaborativa), st3baintang (basadas en intangibles) y st4estrateg (orientadas a la estrategia), presentan valores iguales o menores a 0.15 , lo cual permite aceptar las hipótesis de la investigación, es decir, que las empresas de capital con origen extranjero, se diferencian con niveles más altos en dichos factores, de las locales. El único factor que no discrimina, pues su significancia no se encuentra por debajo de 0.15 , sino que es de 0.34 , es el st 1 proflex (procesos flexibles), lo cual significa que no existe una diferencia, estadísticamente significativa, entre las calificaciones medias de empresas locales y extranjeras, o bien, que esta variable no discrimina y que, por lo tanto, se rechaza la hipótesis. Las posibles razones de este bajo nivel de significancia, se explican en las conclusiones. 


\section{Análisis cualitativo}

Así mismo, se elaboró un cuestionario denominado: cuestionario de relaciones, cuyo objetivo fue determinar si ciertos elementos, denominados pivotantes mueven la evolución de los factores clave, es decir:

a. Las relaciones de confianza contribuyen a tener procesos más flexibles con bajos costos transaccionales.

b. Las organizaciones innovadoras contribuyen a desarrollar procesos más flexibles con menores costos de innovación.

c. Las relaciones de confianza contribuyen a construir organizaciones más innovadoras y basadas en los intangibles con costos más bajos.

d. Las operaciones basadas en tecnologías de la información (TI's) coadyuvan a tener procesos flexibles de bajo costo.

e. Las operaciones basadas en TI's contribuyen a elevar el valor de la organización basada en intangibles.

f. Las organizaciones operando con TI's permiten que el personal efectúe más trabajo estratégico que operativo.

La mayor parte de los reactivos tiene sustento en conceptos y factores justificados en el marco teórico. En el cuestionario de medición, las posibles respuestas son dicotómicas, con calificaciones de cero o cinco basadas en escalas de Likert, con calificaciones en un rango de 0 a 5 , siendo 5 la calificación para las mejores respuestas, según la hipótesis del trabajo y 0 la más baja. Algunos reactivos no eran aplicables para algunas empresas como en los casos de empresas que fabrican partes no visibles de los autos y que por lo tanto, no le conceden ninguna importancia al diseño de sus productos. En otro caso, al ser muy frecuente la respuesta de dato no disponible como el del porcentaje del presupuesto anual destinado a promoción y fortalecimiento de marca, se decidió cancelar el reactivo.

En el cuestionario de relaciones, todas las respuestas están en escala de Likert, que van del total acuerdo (5), de acuerdo (4), indiferencia (3), en desacuerdo (2), hasta el total desacuerdo (1).

Resultados de las hipótesis de relaciones

Al procesar los datos y determinar las medianas de los resultados, estos quedaron dentro de los siguientes rangos:

1-2 Muy bajo

2-3 Bajo

3-4 Alto

4-5 Muy alto

En la Tabla 5 se aprecian los datos condensados por origen de la empresa y por reactivo cuestionado (R), así como también del origen geográfico: 
Tabla 5

Datos condesados por origen de la empresa y por reactivo cuestionado

\begin{tabular}{|c|c|c|c|c|}
\hline & LOCAL & EUR & USA & MEDIANA GRAL. \\
\hline $\begin{array}{l}\text { Relaciones de confianza para } \\
\text { procesos flexibles }\end{array}$ & 4.25 & 4 & 4 & 4 \\
\hline R 1.1 & 4.5 & 4 & 5 & 4.5 \\
\hline R 1.2 & 4 & 2 & 2 & 2 \\
\hline R 1.3 & 5 & 4 & 4 & 4 \\
\hline R 1.4 & 5 & 5 & 5 & 5 \\
\hline R 1.5 & 2.5 & 3 & 2 & 2.5 \\
\hline R 1.6 & 4 & 4 & 4 & 4 \\
\hline $\begin{array}{l}\text { Organización Innovadora } \\
\text { para procesos flexibles }\end{array}$ & 5 & 5 & 5 & 5 \\
\hline R 2.1 & 5 & 5 & 5 & 5 \\
\hline R 2.2 & 5 & 5 & 5 & 5 \\
\hline R 2.3 & 3.5 & 3 & 2 & 3 \\
\hline R 2.4 & 5 & 4 & 5 & 5 \\
\hline R 2.5 & 5 & 5 & 5 & 5 \\
\hline R 2.6 & 5 & 5 & 4 & 5 \\
\hline $\begin{array}{l}\text { Operación basada en TI para } \\
\text { procesos flexibles }\end{array}$ & 4.5 & 4.5 & 4.5 & 4.5 \\
\hline R 3.1 & 5 & 5 & 5 & 5 \\
\hline R 3.2 & 4 & 2 & 2 & 2 \\
\hline R 3.3 & 5 & 5 & 5 & 5 \\
\hline R 3.4 & 4 & 5 & 5 & 5 \\
\hline R 3.5 & 2 & 4 & 4 & 4 \\
\hline R 3.6 & 5 & 4 & 4 & 4 \\
\hline $\begin{array}{l}\text { Relaciones de confianza para } \\
\text { mejorar intangibles }\end{array}$ & 4.0 & 3.5 & 4.0 & 4 \\
\hline R 4.1 & 5 & 3 & 5 & 5 \\
\hline R 4.2 & 4 & 3 & 3 & 3 \\
\hline R 4.3 & 4 & 4 & 3 & 4 \\
\hline R 4.4 & 4 & 3 & 4 & 4 \\
\hline R 4.5 & 4.5 & 4 & 4 & 4 \\
\hline R 4.6 & 4 & 5 & 4 & 4 \\
\hline
\end{tabular}




\begin{tabular}{|l|c|c|c|c|}
\hline $\begin{array}{l}\text { Relaciones de confianza para } \\
\text { fortalecer trabajo estratégico }\end{array}$ & 4.0 & 4.0 & 4.0 & 4 \\
\hline R 5.1 & 4 & 4 & 4 & 4 \\
\hline R 5.2 & 4 & 5 & 4 & 4 \\
\hline R 5.3 & 2 & 2 & 3 & 2 \\
\hline R 5.4 & 3.5 & 2 & 2 & 2 \\
\hline R 5.5 & 4 & 4 & 4 & 4 \\
\hline R 5.6 & 4 & 4 & 4 & 4 \\
\hline Operación basada en TI para \\
mejorar intangibles & 4.3 & 4.0 & 4.0 & 4 \\
\hline R 6.1 & 4 & 4 & 4 & 4 \\
\hline R 6.2 & 4.5 & 4 & 4 & 4 \\
\hline R 6.3 & 4 & 4 & 4 & 4 \\
\hline R 6.4 & 4 & 5 & 4 & 4 \\
\hline R 6.5 & 4.5 & 4 & 4 & 4 \\
\hline R 6.6 & 4.5 & 4 & 4 & 4 \\
\hline
\end{tabular}

Más allá de los resultados obtenidos y analizados, cuantitativamente con herramientas estadísticas, debe considerarse la experiencia de entrevistar a directores y gerentes de las mejores empresas manufactureras de la región dentro del marco de la investigación y con esta los aspectos cualitativos que no figuran como parte del análisis que serán comentados en esta parte del trabajo.

En cuanto al análisis cualitativo de los factores pivotantes, se desprenden los siguientes resultados:

En todos los factores, las medianas son de cuatro o cinco, es decir, altos o muy altos, que permite aceptar la premisa del trabajo, según la cual, los elementos pivote denominados: Relaciones de confianza, operación basada en TI's y organizaciones innovadoras tienen un impacto importante en la gestión de procesos flexibles, mejora de intangibles y el fortalecimiento del trabajo estratégico.

No obstante, cabe destacar que aunque las medianas por grupo de origen de la empresa y generales son altas y muy altas, hubo reactivos en los que los resultados fueron bajos, sin discriminación por origen del capital. Estos son:

R 1.2 Soportar las relaciones, con rígidos contratos legales, contribuye a la producción diversificada con bajos costos transaccionales.

R 1.5 Obtener precios muy bajos y largos plazos de pago con proveedores contribuye a la producción diversificada con bajos costos transaccionales. 
En ambos casos, se esperaban respuestas contrarias, pues en el caso de R 1.2, la literatura que soporta el marco teórico sustenta que las relaciones basadas en rígidos contratos legales las hace lentas, burocráticas y por lo mismo costosas, particularmente, en países en desarrollo como México. Al cuestionar más a fondo a los entrevistados de las razones por las que trabajan de esa forma, la respuesta común, fue que su cliente más importante: Volkswagen de México, es muy difícil y la rotación de compradores de dicha organización y sus cambios constantes de criterios, los obligan a soportar todo por contratos rígidos y bien estipulados, pues no se respetan los acuerdos verbales y cambian las especificaciones y condiciones en forma arbitraria. Los que conocen las relaciones de sus homólogos, en plantas europeas y que llevan la relación con Volkswagen de Alemania, aseguraron que estas son muy diferentes y que allá las relaciones están basadas en la confianza y que la actitud de los compradores y ejecutivos, en Europa, es mucho más colaborativa y amable que la de los mexicanos que trabajan en la planta alemana en Puebla.

En el caso de R 1.5, no hay literatura académica que sustente un argumento sobre los beneficios cuantitativos y cualitativos de pagar de contado a los proveedores, con excepción de una investigación realizada por el autor de esta tesis, en la que se lograron evidenciar, cuantitativamente, los ahorros generados por los descuentos obtenidos por pagar al contado a proveedores, e inclusive, el hecho comprobado de que es más barato financiarse con bancos para pagar de contado a proveedores. El crédito más costoso es el de los proveedores, de acuerdo con tal investigación. Al comentar esta situación al final de la aplicación del cuestionario a los entrevistados, manifestaron desconocimiento de dichos beneficios, asombro, curiosidad e interés por una propuesta tan poco ortodoxa. Sin embargo, para efectos de esta tesis, dicha postura final no cuenta.

R 2.3. Que el personal cubra estrictamente horarios de trabajo, en un espacio físico determinado, contribuye a un sistema diversificado basado en las innovaciones. Aunque no hay mucha literatura académica al respecto, los discursos de expertos y autores como Jack Welch y Tom Peters y el caso del sistema y ambiente de trabajo de Google, sugieren constantemente que la propuesta anterior no hace dicha contribución. No obstante, por los resultados obtenidos, al menos en las empresas instaladas en nuestra región, sin importar su origen, esta propuesta es un paradigma que al parecer no está dispuestos a intentar cambiar.

R 3.2 Tener una organización operando TI's con procesos departamentalizados contribuye a la producción diversificada con bajos costos operativos. A diferencia de los casos anteriores, sí hay bibliografía al respecto; sin embargo, salvo algunos casos excepcionales de empresas de origen local y algunas extranjeras que operan con SAP y admitieron expresamente que trabajar dicho ERP con procesos departamentalizados acota sus funcionalidades, fueron más las que se apegaron al viejo paradigma de los procesos departamentalizados aún después de la implantación de un ERP. Es probable que algunos ni siquiera entiendan bien las diferencias entre organizaciones por funciones $y$ por procesos. 
R 4.2 Implementar estrictos controles de entradas y salidas a personal de confianza contribuye a innovar con menor esfuerzo y en menos tiempo. Es la misma situación que en el reactivo $\mathrm{R} 2.3$ y del $R$ 5.4. Las tres tuvieron medianas de tres a menos. Hubo consistencia en las tres respuestas. Sin embargo, cabe mencionar que las empresas locales tuvieron mejores respuestas en los tres reactivos.

R 5.3 Permitir acceso exclusivo a información financiera y de mercado a las áreas correspondientes contribuye al crecimiento del negocio con costos más bajos.

En general, se aprecia renuencia en factores en los que el personal tenga mayor libertad de horarios y espacios físicos, igualmente en compartir información con terceros, así como reducir tiempos de pagos a proveedores. Aunque en las entrevistas, en cuanto a los procesos departamentalizados mostraban dudas sobre el concepto, en cuanto a los beneficios de una actitud más colaborativa al pagar pronto a proveedores, demostraron desconocimiento de sus beneficios, algo que puede parecer natural, pues es uno de los paradigmas más frecuentes en las operaciones de compras en las empresas, y que de hecho, son pocos los que han evidenciado los beneficios económicos y en términos de colaboración y relaciones de confianza.

\section{Conclusiones}

Las empresas locales seleccionadas son lo mejor de la región como se puede ver. Todas exportan y ello las obliga a ser más competitivas. Algunas son proveedoras del sector automotriz, como varias de las extranjeras, así son parte de la compleja y exigente dinámica de la cadena de suministros del sector automotriz y en uno de los casos, de Wal-Mart. Otras tienen socios estratégicos de países desarrollados, que en muchos casos, las condicionan a la implantación de las mejores prácticas como parte de la sociedad; aunque lo siguiente no aparece como un dato cuantitativo, en la investigación, como resultado de conocer a los entrevistados, quienes en su mayoría tienen estudios avanzados de Ingeniería y negocios, y en el único caso en que no es así, el Director cuenta con equipos gerenciales formados por profesionales jóvenes, y la región cuenta con una importante presencia de instituciones educativas de alto nivel. Eso se refleja en la competitividad de las empresas manufactureras como lo evidencia esta investigación en el factor de los procesos flexibles, que depende más de la inversión en tecnología y de la aplicación sistemática y disciplinada de las ampliamente reconocidas y difundidas prácticas japonesas en el piso de producción. Todo esto puede explicar que, al menos en dicho factor no presenten un rezago significativo respecto de las empresas globales instaladas en la región.

Con lo mencionado, anteriormente, a partir de nuestro cuestionario quedó aceptada tres de cuatro factores. Sin embargo, hubo reactivos específicos de cada factor que marcaron diferencias significativas aún dentro del mismo factor menos significativo, el de procesos flexibles. Al medir la significancia por factor, se pudieron apreciar las diferencias en los siguientes reactivos:

En el factor procesos flexibles, los únicos elementos que tuvieron una variación 
significativa entre extranjeras y locales basadas en prueba U Mann Whitney, fueron:

Porcentaje de proveedores en desarrollo $(\mathrm{P}=0.01)$. Aunque es un elemento muy relacionado con el factor de competitividad colaborativa, se incluyó en el factor de procesos flexibles, pues es un inductor de dicha flexibilidad. Esto refuerza también la hipótesis aceptada de las diferencias significativas en competitividad colaborativa entre empresas extranjeras y locales.

Grado de integración regional de proveeduría $(P=0.01)$. En el caso de este elemento, sí discrimina, pero a favor de las empresas locales, que mostraron una base de proveedores locales más elevada que las extranjeras. Puede parecer lógico que sean las empresas locales las que busquen proveedores nacionales más que las extranjeras, que suelen tener contratos corporativos globales de abastecimiento, incluso con proveedores de sus países de origen a pesar de las distancias.

Porcentaje de incorporación de pedidos de clientes directamente por ERP/ CRM $(\mathrm{P}=0.05)$. Particularmente, las proveedoras extranjeras de autopartes tienen esta práctica obligada con sus clientes.

\section{Referencias}

Beinhocker, E. (2007). The origin of wealth. Harvard Business School Press.

Charles, H., and Gareth, R. (2001). Strategic Management. Houghton Mifflin.

Cheung, Y. (2006). Supply chain sustainability - the role of trust and relationship. Faculty of Built Environment and Engineering, Queensland University of Technology.

Contractor, F.J., and Lorange, P. (2002). The growth of alliances in the knowledge based economy. International Business Review. V. 11. 485-502.

Dussel, E. (2006). Hacia una política de competitividad en México. Economía UNAM, (3)9.
Evans, P. and Wolf, B. (2005). Collaboration Rules. Harvard Business Review. July-August.

Fuentes E. (1996). Alianzas Estratégicas. México: Dirección Ejecutiva de desarrollo de servicios promocionales.

Fung, V., Fung, W., and Wind, Y. (2008). Competing in a flat world. Wharton School Publishing.

Futuyma, D. (2005). Evolution. Sinauer Associates, Inc.

Gratton, L. (2007). HOT SPOTS Why some teams, workplaces, and organizations buzz with energy and others don't. Berret Koehler Publishers Inc. 
Handfield, R. and Bechtel, C. (2002). The role of trust and relationship structure in improving supply chain responsiveness. Industrial Marketing Management 31 (2002) 367-382.

Harrison, L. \& Huntington, S. (2000). Culture Matters: How Values Shape Human Progress. New York: Basic Books.

Japan Institute of Plant Maintenance. (1996). TPM for Every Operator. Productivity Press.

Journal of memetics (http://www.jomemit.org)

Landes, D. (2008). La riqueza y la pobreza de las naciones. Editorial Crítica.

IMCO. (2007). Punto de Inflexión. Situación de la competitividad en México 2006. IMCO.

Laseter, T. (2000). Alianzas estratégicas con proveedores. Grupo Ed. Norma.

Liker, J. (2003). The Toyota Way: 14 Management Principles from the World's Greatest Manufacturer. First Edition. McGraw-Hill

Lowell, B. and Joyce, C. (2007). Mobilizing minds. McGraw Hill.

McSpadden, W. (2001). DIRECT from DELL.” (Interview). Technology Review. Cambridge, Mass. July 2001. vol. 104, i 6, p. 78.

Osterwalder, A. (2004). The Business Model Ontology - A Proposition In A Design Science Approach. Thesis by Alexander Osterwalder.
Pill, Huw. (2002). The US in 2001: Macroeconomic policy and the new economy. Harvard Business School Publishing.

Porter, M. and Stern, S. (1999). The new Challenger to America's prosperity. Findings from the innovation index. Council on Competitiveness.

Pragman, C. (1996). JIT II: a purchasing concept for reducing lead times in time-based competition. (Justin-time management). Business Horizons. July-August 1996.

Scotchmer, A. (2008). 5 S Kaizen in 90 Minutes. Management Books 2000 Ltd.

Shingo, S. (1985). Zero quality control: source inspection and the poka-yoke system. Productivity Press.

Syeda, N., and Syeda A. (2008). Trust in buyer-supplier relations: the case of the Turkish automotive industry. Journal of International Business Studies. 39, 118-131.

Vangen, S. and Huxham, C. (2003). Nurturing Collaborative Relations: building trust in interorganizational collaboration. Journal of Applied Behavioral Science. 2003; 39; 5

Zaheer A., McEvily B. \& Perrone V. (1998). Does trust matter? Exploring the effects of interorganizational and interpersonal trust on performance. Organization Science 1998; 9 (2): 141-159. 
